\title{
OpenBuild : An Integrated Simulation Environment for Building Control
}

\author{
Tomasz T. Gorecki ${ }^{1}$ and Faran A. Qureshi ${ }^{1}$ and Colin N. Jones ${ }^{1}$
}

\begin{abstract}
This paper introduces the OpenBuild toolbox for MATLAB. OpenBuild is a toolbox for advanced controller design for buildings heating ventilation and air conditioning systems, with emphasis on Model Predictive Control. It provides researchers in the control community the ability to test algorithms on a wide range of realistic simulation scenarios, by providing most of the data needed to perform simulation and optimization. It combines the convenience of controller design in MATLAB with the simulation capabilities of the building simulation software EnergyPlus. It includes a building modeling tool to construct linear state-space models of building thermodynamics based on building description data, making it useful for design of optimal controllers requiring a good prediction model, as well as providing the input data necessary for simulation such as weather, occupancy and internal gains data. The ability to co-simulate the building between MATLAB and EnergyPlus enables fast prototyping and validation of the models and controllers. This paper presents the working principles and functionality of OpenBuild.
\end{abstract}

\section{INTRODUCTION}

In addition to ocuupants comfort and indoor air quality, energy efficiency is a primary target in building climate control. Model Predictive Control (MPC) has been demonstrated to be a competitive control algorithm for building energy systems [1]. Its advantages include the ability to explicitly deal with constraints, incorporate predictions and optimize complex economic objectives. Research on MPC for building control is moving towards more complex applications such as demand response potential assessment [2] where the advantages of using optimal controller become even more apparent. One of the main difficulties of MPC design for buildings is to create and validate models capturing the behavior of the building and the Heating, Ventilation and Air Conditioning System (HVAC) and are appropriate for controller design. Proper validation of control algorithms requires extensive testing on a variety of typical weather scenarios, building types and usage, therefore requiring timeconsuming efforts to create databases of simulation scenarios and building models [3], [4].

A wide range of simulation software exists for buildings, such as TRNSYS and EnergyPlus. EnergyPlus [5] focuses on thermodynamics of the building and HVAC simulation, and has been extensively validated [6]. Like most simulation

\footnotetext{
${ }^{1}$ The authors are with the Laboratory of Automatic Control in EPFL, Lausanne, Switzerland. \{tomasz.gorecki, faran.qureshi,colin.jones\} @epfl.ch. This work has received support from the Swiss National Science Foundation under the GEMS project (Green Energy Management of Structures, grant number 200021 137985) and the European Research Council under the European Unions Seventh Framework Programme (FP/2007-2013), ERC Grant Agreement 307608.
}

software, it is however not aimed at advanced controller design. It has been pointed out that efforts have to be made to enable easier co-simulation and controller design for buildings [7]. The interfaces BCVTB [8] and MLE+ [9] have been developed to allow interfacing of MATLAB and EnergyPlus, enabling easier development and simulation of controllers. However, these tools require knowledge of EnergyPlus, and do not address the issue of modeling.

Obtaining controller-ready models remains the main hurdle for MPC controller design for buildings. [10] uses EnergyPlus as the prediction engine of MPC controllers. It allows the control of variables externally interfaced in EnergyPlus without extensive knowledge of the underlying system, but imposes the use of black-box nonsmooth optimization techniques such as particle swarms algorithms, which can be very slow. In [11], a linear model taking HVAC setpoints and occupancy levels as inputs and energy usage as output is identified based on simulation data from EnergyPlus automatically. Nevertheless, the simple models obtained do not capture all factors influencing the building, e.g., weather. Most of the literature of MPC for buildings relies on statespace, often linear representations of buildings in order to use classical optimization tools. The toolbox BRCM [12] helps to create discrete-time state-space models for buildings using a physical modeling approach. It can partly use EnergyPlus building description files as input files but requires further inputs from the users. However, it does not provide input data compatible with the model for weather and usage description, and does not offer co-simulation capabilities.

The OpenBuild toolbox is a modeling and co-simulation tool created to design, test and validate controllers in simulation for building systems. It provides a workflow with all elements required to perform simulation of complex controllers in realistic scenarios. One of the key components of the toolbox is the system modeler, taking standard building description data files. It creates automatically linear statespace models for the thermodynamics of the building and collects disturbance input data for the effect of the weather and internal gains. This feature provides control researchers with models and data which are ready to use and suitable for MPC controllers, thus enabling simulations of complex scenarios with real weather patterns and occupancy data at a limited effort. This makes OpenBuild suited for MPC design. The software includes MLE+ for co-simulation between MATLAB and EnergyPlus which helps validating models and testing controllers easily. In addition, EnergyPlus is a validated and trusted source of information for building models and data. A database of validated building models 
for EnergyPlus is available [13] as well as a large range of weather description files. For simplicity, HVAC modeling can be done in MATLAB, requiring almost no knowledge of EnergyPlus inner workings and external interface mechanisms. Heat fluxes to the rooms in co-simulation can directly be controlled in MATLAB, allowing the user to model additional modules attached to the building in MATLAB. OpenBuild allows one, for instance, to easily simulate the addition of batteries, heat storage or photovoltaic panels attached to the building in MATLAB. The potential of this tool has been demonstrated by performing advanced simulations of Demand Response participations [2].

Section II outlines the main components of the toolbox. Section III goes through the main modeling principles used in the software. Section IV presents applications which illustrate the capability of the software, with model validation and design of an advanced MPC controller for thermal storage control.

\section{THE SIMULATION WORKFLOW IN OPENBUILD}

The main objective of OpenBuild is to enable the design and testing of advanced controllers, especially MPC controllers, in realistic simulation scenarios. It builds on the cosimulation interface MLE+ to provide control experts most of the tools and data required for controller design for buildings, as pictured in Figure 1. The OpenBuild toolbox helps to collect and construct these components, and streamlines their use in an integrated workflow.

Co-simulation is considered a valuable option for control design [8]. EnergyPlus is a widely used high-fidelity simulation environment, but it is not suited for complex controller design. Recent contributions [9] have enabled cosimulations between EnergyPlus and MATLAB, where the controller is designed and simulated in MATLAB. However, co-simulations require a number of other elements including building description data, description of weather, occupancy and usage of the building. MPC design requires models of the building and HVAC system suitable for optimization. Simulations also require the conversion of weather and occupancy data to the actual inputs of the models used for control. This section details each of these components as they are included in OpenBuild. Figure 1 summarizes the different modules of OpenBuild with letter labels referring to the following subsections.

\section{A. Building and weather data}

Relying on EnergyPlus allows to directly use existing description data for buildings, such as the DOE Reference buildings set [13]. In addition, other tools [14] help users to easily create new models for EnergyPlus. Lastly, conversion from other building description formats is often possible. For usage description, EnergyPlus input data files include schedules of occupancy, equipments, lights, etc. that OpenBuild can directly interpret.

EnergyPlus takes standard weather data files as input. Typical weather data for numerous locations is readily available.
Moreover, using EnergyPlus utility programs, additional weather files can be created based on measured or forecast weather data, also reconstructing missing or corrupted data. This is extremely useful to construct predictions of the weather disturbances for an MPC controller.

\section{B. Thermodynamics simulator}

EnergyPlus can be used as the simulator for the thermodynamics of the building. It is possible to control some variables in EnergyPlus through an external interface, and [9] provides ways to run co-simulations from MATLAB. However, two main difficulties arise: first the external interface lacks flexibility and requires knowledge of EnergyPlus and manual modification of the files. Second, only specific variables are available for external control, mostly setpoints for thermostats. For most systems, no direct control of the low-level actuators and variables is possible (valve and damper positions, massflows, etc.). This issue is common to numerous building simulation software, which are generally not well suited for controller design. Therefore, to enable flexible HVAC simulation, OpenBuild uses EnergyPlus only for the thermodynamics of the building. From MATLAB's point of view, the inputs to the zones are heat fluxes to the rooms or surfaces of the building. This allows the decoupling of the simulation of the building and the HVAC. This is a reasonable setup since the thermodynamics of the building is mostly independent from the HVAC type.

Remark 1: The models generated by OpenBuild can also be used to simulate the building in MATLAB without cosimulation.

\section{HVAC simulator}

Modeling of the HVAC is a complex task, which is very difficult to perform automatically. The complexity of the HVAC descriptions in EnergyPlus are high, at a level of detail which is not required for controller design. Most works from the literature report targeted case-by-case modeling efforts for the HVAC, which is very time-consuming. In the perspective of large-scale simulations of building controllers, this motivates the use of HVAC models in MATLAB. These models map the actual input (such as electric power input, valve and damper positions or fluid flows) to the heat fluxes into the different rooms and surfaces. A framework is proposed to specify new HVAC system models easily. Some simple HVAC models have been developed and include simple forced-air systems, thermally activated building systems, electric boilers, heat pumps, and blind controls. In addition to simulating the HVAC, the HVAC simulator also computes appropriate inputs to the external interface of EnergyPlus. Additional modules such as batteries or storage tanks can easily be added and simulated together with the building. Notice that HVAC components can still be simulated in EnergyPlus in co-simulation but that requires manual processing of the files and good knowledge of EnergyPlus inner workings. 
Fig. 1. Dataflow in OpenBuild co-simulations

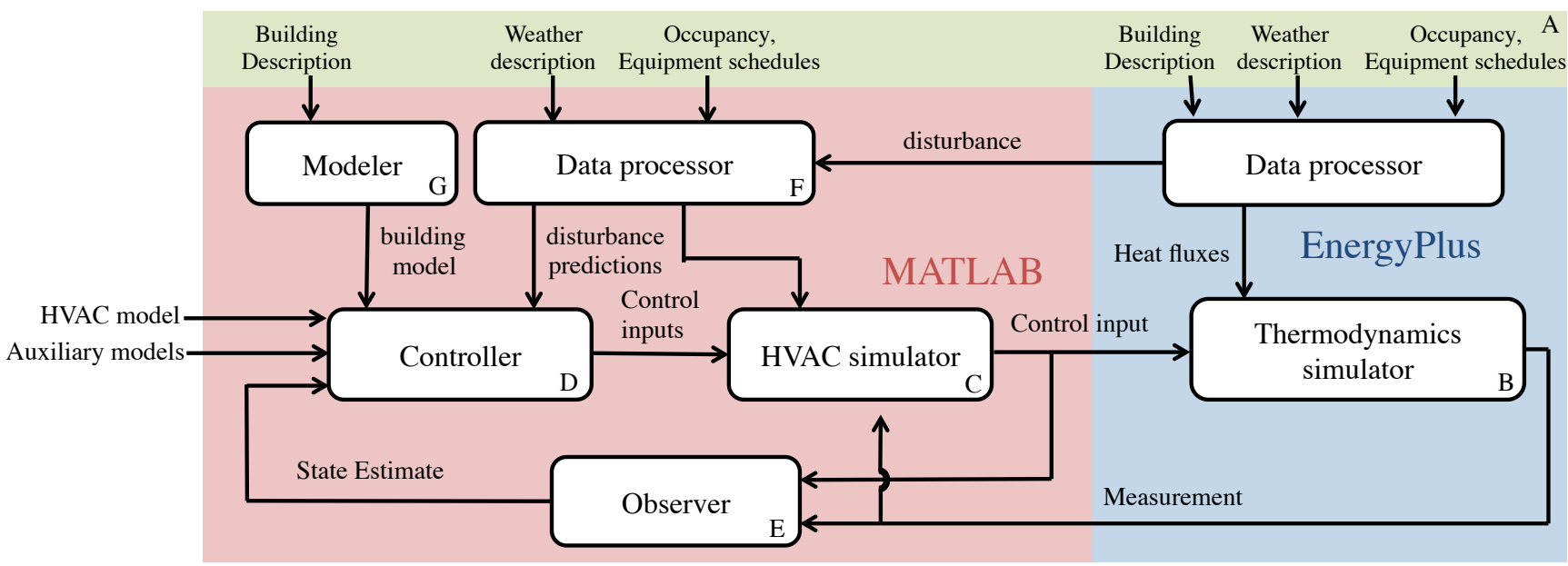

\section{Controller}

Good controllers are imperative for the efficient operation of a building. OpenBuild focuses on MPC controllers. The controllers use a model of the dynamics of the system and solve a constrained optimization problems to compute an optimal input sequence. The performance of MPC controller relies greatly on the quality of the model. Openbuild can directly extract models for the thermodynamics of the building ( $c f$ Section II-G) combined with the models of the HVAC system and auxiliary systems simulated in MATLAB. MPC is particularly suitable for flexible objective specifications through the cost function and constraints. The temperatures are generally constrained to lie in possibly timevarying bounds. Objectives such as minimum energy use and minimum cost are usual. It can prove particularly efficient in the presence of time-of-use electricity tariff schemes, and can enable the control of buildings for participation in complicated demand response programs [2]. An environment is provided in the toolbox which facilitates MPC controller design. Classical MPC formulations such as soft constraints and offset-free formulations [15] are easily implemented and available in the examples of the toolbox.

\section{E. Observer}

Full state information of the linear model is required for control with MPC, however it is not available from EnergyPlus (or in a real building). Observers are required to estimate the state of the building, HVAC system, and auxiliary systems attached to it. Observer design can be challenging because of model mismatch and disturbance issues. By combining the offset-free formulation [15] and Kalman filtering, good performance has generally been achieved. The Kalman filter is also designed using the model of the building. Examples of filters and controllers are available in the toolbox examples but tuning of the observers has been observed to have a significant impact on the quality of the estimation, therefore requiring a minimum effort from the user.

\section{F. Data Processor}

Implementation of MPC controllers requires the prediction of the weather, including solar gains, occupancy, and other internal gains. Occupancy and equipment use are usually specified under the form of schedules directly in the EnergyPlus format. Weather data comes in separate files which list temperatures, humidity ratios, weather conditions, solar irradiance, etc. This data needs to be interpreted to evaluate the impact of the weather on the building, e.g. through geometric computations to calculate the effect of sun on each surface. EnergyPlus performs these computations, which we can directly exploit in OpenBuild. Openbuild uses EnergyPlus as a preprocessing engine for the model. From just the building and weather description, it automatically runs the appropriate components of EnergyPlus to extract the corresponding weather and internal gain data compatible with the models. This is a key feature of OpenBuild which facilitates simulation greatly by requiring minimum user input.

\section{G. Modeler}

When running simulations, EnergyPlus uses standard input files, describing the geometry and construction of the building, the heating system and simulation parameters. Based on the information in these files, it computes other quantities for the simulation, such as equivalent $U$-values of windows, viewing factors of internal surfaces, etc. This processed data is given out as output of simulations with EnergyPlus. OpenBuild automatically generates a linear state-space model of the building thermodynamics based on the input data files and the processed data from EnergyPlus. This automatic model generator is the backbone of the OpenBuild toolbox. Detailed description of the modeling procedure is given in Section III.

\section{MODELING PROCEDURE}

The goal of the modeling procedure is to obtain a model which is simple enough to be suitable for control (especially MPC), yet capturing satisfactorily the dynamics of the building. A physical modeling approach is adopted. The following physical phenomena are modeled: 
- Heat transfer through conduction

- Heat transfer through convection

- Long-wave radiation on all internal and external surfaces

- Internal gains (lighting, occupancy, equipment) on all internal surfaces

- Solar radiation on internal and external surfaces

\section{A. Modeling Fundamentals}

The well-established RC modeling framework [16], [17] is used to model the thermodynamics of the building. It consists in representing the building as a set of thermal nodes in a graph where the temperature dynamics of each node is described by a linear differential equation. A parallel with electrical circuits illustrates best the concepts: temperatures of the zone air and of the building elements are represented by the voltages at each node of the RC network. Heat flux transfer between the lumped parameters of the building are equivalent to currents between nodes of the RC network. Coefficients of heat conduction between the nodes and convection between the zone air and the building surfaces are modeled by resistances in the RC network. The thermal capacity of the zone air and of the layers in the building surfaces are modeled by the capacitors. Long wave radiation from outside and between surfaces are also linearized and represented by resistances.

\section{B. Model Parameters}

The computation of the parameters in the $\mathrm{RC}$ model is carried out using both the input data file and the post processed EnergyPlus data (surface view factors, convection coefficients, etc.). The thermal capacity of the lumped parameters and the conduction coefficients in the RC model depend on the physical properties of the material used in the building construction, as described in the building data file. The convection coefficients in the RC model depend on the material properties, but also on other external factors including weather conditions. In EnergyPlus, the computation of convection coefficients can be carried out using different algorithms (see [18], pp.64-74, 78-94), and yields time-varying convection coefficients. A constant time averaged coefficient is considered in the model extraction and is collected from the post-processed EnergyPlus data. The long-wave radiation from the external sources and between the internal surfaces of the building is characterized by a nonlinear function (see [18], pp. 76-77). This function is linearized, viewing factors are obtained from the post-processed EnergyPlus data and the physical properties of the construction material are obtained from the building data file. The solar radiation and the internal gains acting on the building surfaces are obtained from the post-processed EnergyPlus data and are applied to the corresponding nodes of the RC network. Lastly, EnergyPlus computes equivalent U-values capturing the overall heat transfer through windows, which are used by OpenBuild for window modeling.

\section{Model Structure}

The following energy flux balance equation is applied at each node of the RC model:

$$
C_{n} \frac{d T_{n}}{d t}=Q_{c}+Q_{g}+Q_{r}+Q_{\mathrm{HVAC}}
$$

where $C_{n}$ is the thermal capacity and $T_{n}$ is the temperature of node $n$, respectively. $Q_{c}$ combines the heat flux acting on the node due to conduction and convection, $Q_{g}$ is the flux from solar and internal gains, $Q_{r}$ is the flux due to radiation, and $Q_{\mathrm{HVAC}}$ is the flux from HVAC acting on the node. This results in a set of linear differential equations. The windows are a special case in the model, since they don't have any thermal capacity: they are modeled by a set of algebraic equations (see [18], pp. 225-231). We use a linearized version of these equations to obtain explicit expressions of the window surface temperatures and substitute it in the differential equations of the rest of the temperature nodes.

This procedure provides a linear state-space model of the building which is discretized to obtain a model of the form:

$$
\begin{aligned}
x_{k+1} & =A x_{k}+B_{u} u_{k}+B_{d} d_{k} \\
y_{k} & =C x_{k}
\end{aligned}
$$

where $x_{k} \in \mathbf{R}^{n}$ is the state vector (containing the temperatures of all the zones, surfaces, and internal nodes), $u_{k} \in \mathbf{R}^{n_{u}}$ is the control input ( $\left.Q_{\mathrm{HVAC}}\right)$, and $d_{k} \in \mathbf{R}^{n_{d}}$ is the weather (e.g., outside temperature and solar gains) and internal gains disturbance vector.

Remark 2: The complete modeling procedure described in this section from creating an RC network graph, computation of the model parameters, solving of algebraic equations, to obtaining the linear model (2) is carried out automatically, taking as input only the building data description file and the weather description file.

\section{APPLICATIONS}

\section{A. Validation of model}

We present an example of co-simulations performed for a building taken from the EnergyPlus examples. The building is a single story office building with three zones. It is simulated for a period of two weeks in winter with typical equipment and occupancy schedules. A minimum energy controller is implemented with night setbacks. The control inputs are the heat fluxes to each zone and a minimum energy consumption is taken as the objective of the MPC problem. Soft comfort constraints are implemented and an offset-free formulation is used.

The input and disturbance profiles computed in this first simulation are taken as open loop input profiles to the linear state-space model constructed by OpenBuild. The resulting temperatures for one zone is depicted in Figure 2. The linear model captures the variations in temperatures in response to changes in the heating level, solar irradiance, and internal gains. Over the simulation period the RMSE error is $1.12^{\circ} \mathrm{C}$ and the maximum error is $2.15^{\circ} \mathrm{C}$. Simulations suggest that the quality of the predictions depends on the external weather conditions. Particularly, prediction quality decreases when 


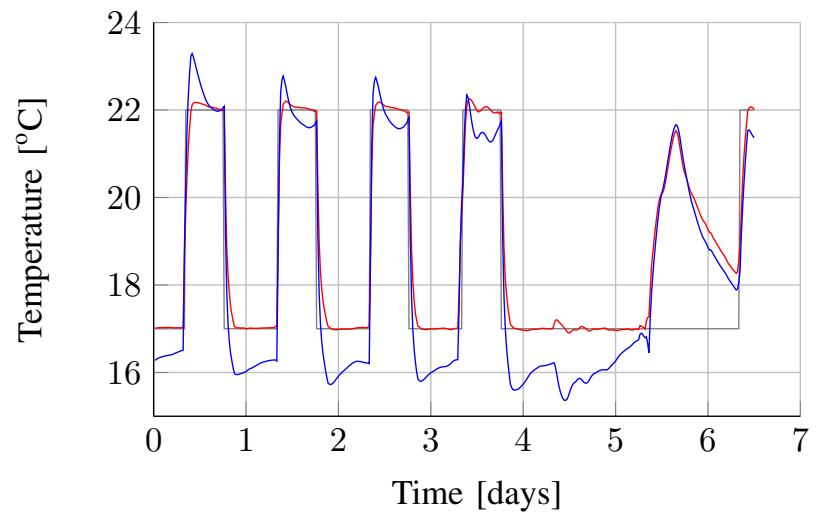

Fig. 2. Temperature in one rooms from EnergyPlus simulation (red) and linear model with the same inputs (blue). Grey line shows the min. temperature constraint.

solar radiation levels are high. Overall, models built with OpenBuild give very satisfying MPC performance provided an appropriate offset-free formulation is adopted and proper observer tuning is achieved. Despite possible parameter errors, the laws of energy conservation are respected, which leads to a model that is always stable and displays physically consistent dynamics.

Remark 3: Several research works have focused on identification of some parameters of the linear model representing buildings. Implementing these identification procedures can be greatly helped by using the automatically generated graph representation of the building model from OpenBuild.

\section{B. Control of thermal storage through MPC}

We consider a large twelve storey office building located in New-York taken from the DOE Commercial Building Reference set [13]. The building has 19 zones served by a forced air heating and cooling system. We focus in this example on the use of a thermal storage for load shifting and minimizing the total cost of building operation. We assume the building has a cold water tank which is supplied by an electrical heat pump. A step-by-step procedure to carry out this simulation using OpenBuild is presented, outlining how the toolbox helps the user performing the tasks easily.

Step 1: A building object is initialized using as input the building data file and the weather data file. All required data is imported to MATLAB. During this process, EnergyPlus is first run once through Openbuild and the processed data from the simulation is collected.

Step 2: The building data is used to automatically generate a linear state-space model of the form of equation (2). At this point the inputs to the model are heat fluxes to each zone. For simplicity, it is considered here that each zone is served by an individual air handling unit which controls the heat flux to the room. The total cooling load of the building is given by $q_{\text {load }}=\sum_{k=1}^{n_{u}} u_{k}$ where $u_{k}$ is the heat power input to zone $k$.

Step 3: A simulation engine object is initialized. This object handles the communication between the different objects simulated, either in MATLAB or in EnergyPlus. EnergyPlus is added as a simulator for the thermodynamics.
Step 4: A cold water tank is modeled in MATLAB and added to the simulation engine object. The tank is assumed to be perfectly stirred and the heat pump has a fixed coefficient of performance. Therefore, the tank dynamics model takes a very simple form:

$$
C_{p} V \dot{T}_{t k}=\alpha\left(T_{r}-T_{t k}\right)-\eta_{c} P_{e}+q_{\text {load }}
$$

where $T_{t k}$ is the temperature of the cold water tank which stands in a room with constant temperature $T_{r} . C_{p}$ is the heat capacity of water, $V$ is the volume of the tank, and $\alpha$ is a coefficient representing heat leakage out of the tank. $\eta_{c}$ is the coefficient of performance of the heat pump and $P_{e}$ is the electrical power consumption of the heat pump. This model has been created manually, and it is then added to the simulation engine automatically.

Step 5: This is the main step where user input is normally necessary. The user needs to implement a controller in MATLAB, possibly using the building model constructed by OpenBuild. In our case, the building model is discretized with a time-step of 30 minutes and is reduced using the Hankel-Norm based balanced truncation method. The resulting model is used as the prediction model along with the storage tank model in an MPC controller. The MPC controller is designed to minimize the total cost of operation in the presence of day-night electricity tariffs. An offset-free formulation [15] with soft comfort constraints is implemented. Night and weekend setbacks (time varying constraints) on the zone temperature are used. A prediction horizon of one day is considered. The following constraints are applied:

$$
\begin{array}{r}
0 \leq P_{e} \leq P_{\max } \\
u_{k, \min } \leq u_{k} \leq u_{k, \max } \\
T_{\min } \leq T_{t k} \leq T_{\max }
\end{array}
$$

where $P_{\max }, u_{k, \min }$ and $u_{k, \max }$ are the maximum electrical power for the heat pump, and the minimum and maximal inputs, respectively. $T_{\min }$ and $T_{\max }$ represent minimum and maximal allowed temperatures in the storage tank.

Step 6: The models of the building and the storage are used to design the observer.

Step 7: Finally, the simulation engine runs the closed-loop simulation and the simulation data is saved. The simulation is run for a period of one week during the summer of 2012, using the real weather data of New York.

Readers are referred to the OpenBuild manual [19] for a comprehensive description of the toolbox use.

Simulations are performed for different sizes of the storage tank and the total electricity consumption of the building over a period of one week is compared. The results are depicted in Figure 3. As seen in this figure, the percentage reduction in the total cost of electricity consumption compared to the case with no storage tank, increases with the size of the storage tank. This is explained by the capability of the building to shift its electricity consumption to off-peak periods using the storage tank. The cumulative energy consumption over a period of one week for the case with no storage tank and with 
Fig. 3. Percentage decrease in the total cost with varying size of storage

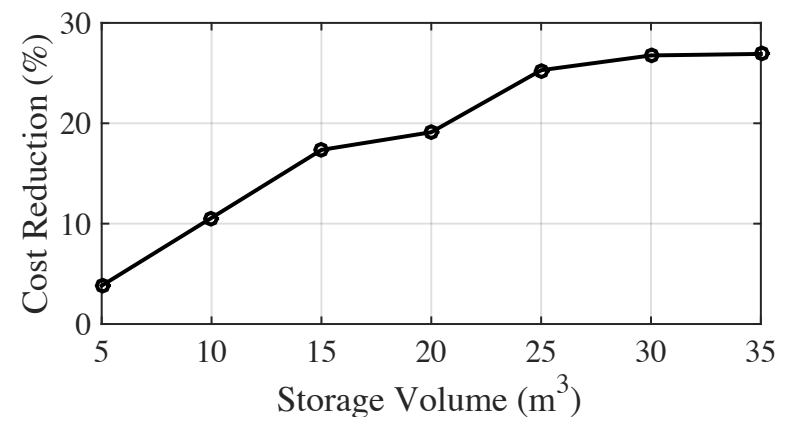

Fig. 4. Impact of storage $\left(30 \mathrm{~m}^{3}\right)$ on building's cumulative energy use

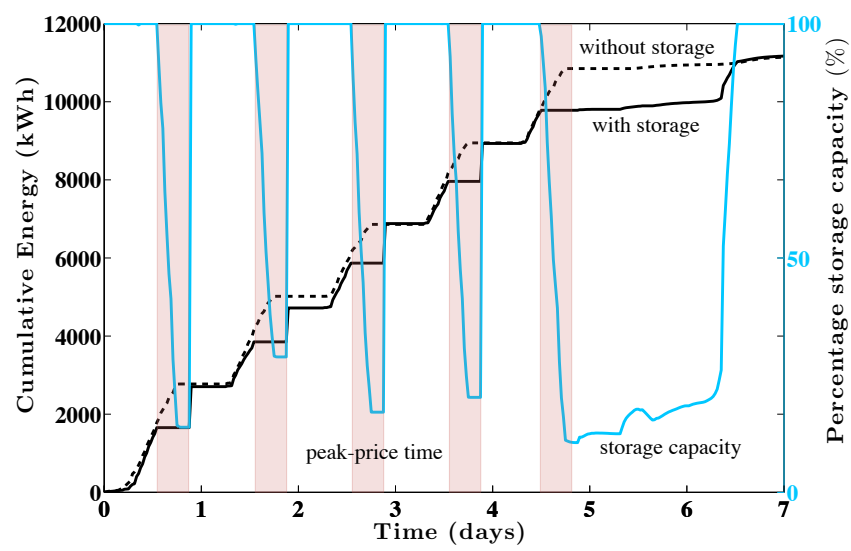

a $30 \mathrm{~m}^{3}$ storage tank is shown in Figure 4. The high tariff price periods have a shaded background. With the storage tank, the cumulative electrical energy consumption is constant while there is a sharp rise just after the peak electricity price period. Without storage the building consumes more electricity during the higher price period, increasing the total cost of operation.

\section{CONCLUSION}

OpenBuild has been designed to offer an integrated environment allowing one to take benefit from the convenience and power of the computing environment MATLAB for controller design and the accuracy and extensivity of the simulation software EnergyPlus for building thermodynamics and HVAC simulation. It is targeted at control engineers or researchers wishing to design and test controllers on realistic building examples through co-simulation with a limited effort. The use of EnergyPlus gives access to large validated datasets for building and weather description that allow to simulate a variety of buildings.

\section{APPENDIX}

The OpenBuild MATLAB toolbox is distributed under the GPL license and is available for download at http: / / la . epfl.ch/openBuild.

\section{ACKNOWLEDGMENT}

Other softwares have been included in the OpenBuild software. These include the Openstudio Ruby SDK, the MLE+ toolbox, the BCVTB interface, and the mksqlite toolbox for MATLAB.

\section{REFERENCES}

[1] Y. Ma, A. Kelman, A. Daly, and F. Borrelli, "Predictive Control for Energy Efficient Buildings with Thermal Storage: Modeling, Stimulation, and Experiments," IEEE Control Systems, vol. 32, no. 1, pp. $44-64$, Feb. 2012.

[2] F. A. Qureshi, T. T. Gorecki, and C. Jones, "Model predictive control for market-based demand response participation," in 19th World Congress of the International Federation of Automatic Control, 2014.

[3] F. Oldewurtel, A. Parisio, C. N. Jones, D. Gyalistras, M. Gwerder, V. Stauch, B. Lehmann, and M. Morari, "Use of model predictive control and weather forecasts for energy efficient building climate control," Energy and Buildings, vol. 45, pp. 15-27, 2012.

[4] G. P. Henze, A. R. Florita, M. J. Brandemuehl, C. Felsmann, and H. Cheng, "Advances in Near-Optimal Control of Passive Building Thermal Storage," Journal of Solar Energy Engineering, vol. 132, no. 2, p. 021009, 2010.

[5] D. B. Crawley, L. K. Lawrie, F. C. Winkelmann, W. Buhl, Y. Huang, C. O. Pedersen, R. K. Strand, R. J. Liesen, D. E. Fisher, M. J. Witte, and J. Glazer, "EnergyPlus: creating a new-generation building energy simulation program," Energy and Buildings, vol. 33, no. 4, pp. 319331, 2001.

[6] R. H. Henninger, M. J. Witte, and D. B. Crawley, "Analytical and comparative testing of EnergyPlus using IEA HVAC BESTEST E100E200 test suite," Energy and Buildings, vol. 36, no. 8, pp. 855-863, Aug. 2004.

[7] C. Sagerschnig, D. Gyalistras, A. Seerig, S. Privara, and Z. Vana, "Co-simulation for building controller development: the case study of a modern office building," in Proceedings CISBAT, 2011, pp. 955-960.

[8] M. Wetter, "Co-Simulation of Building Energy and Control Systems with the Building Controls Virtual Test Bed," Journal of Building Performance Simulation, no. August, 2011.

[9] W. Bernal, M. Behl, T. X. Nghiem, and R. Mangharam, "MLE + : A Tool for Integrated Design and Deployment of Energy Efficient Building Controls," in Proceedings of the Fourth ACM Workshop on Embedded Sensing Systems for Energy-Efficiency in Buildings, 2012, pp. $123-130$.

[10] C. D. Corbin, G. P. Henze, and P. May-Ostendorp, "A model predictive control optimization environment for real-time commercial building application," Journal of Building Performance Simulation, vol. 6, no. 3, pp. 159-174, 2013.

[11] W. J. Cole, E. T. Hale, and T. F. Edgar, "Building energy model reduction for model predictive control using OpenStudio," in Proceedings of the 2013 American Control Conference, Washington, DC, USA, 2013, pp. 449-454.

[12] D. Sturzenegger, D. Gyalistras, V. Semeraro, M. Morari, and R. S. Smith, "Brcm matlab toolbox: Model generation for model predictive building control," in American Control Conference (ACC), 2014. IEEE, 2014, pp. 1063-1069.

[13] US Department of Energy: Office of Energy Efficiency and Renewable Energy, "Commercial Reference Buildings." [Online]. Available: http://energy.gov/eere/buildings/commercial-reference-buildings

[14] R. Guglielmetti, D. Macumber, and N. Long, "OpenStudio: an open source integrated analysis platform," in Proceedings of the 12th Conference of International Building Performance Simulation Association, 2011.

[15] U. Maeder, F. Borrelli, and M. Morari, "Linear offset-free Model Predictive Control," Automatica, vol. 45, no. 10, pp. 2214-2222, Oct. 2009.

[16] D. Sturzenegger, D. Gyalistras, M. Morari, and R. S. Smith, "Semiautomated modular modeling of buildings for model predictive control," in Proceedings of the Fourth ACM Workshop on Embedded Sensing Systems for Energy-Efficiency in Buildings, ser. BuildSys '12. New York, NY, USA: ACM, 2012, pp. 99-106.

[17] Y. Lin, T. Middelkoop, and P. Barooah, "Issues in identification of control-oriented thermal models of zones in multi-zone buildings," in 2012 IEEE 51st Annual Conference on Decision and Control (CDC), Dec. 2012, pp. 6932-6937.

[18] U. DOE, EnergyPlus Engineering Reference, 2012.

[19] T. T. Gorecki and F. A. Qureshi, "OpenBuild Manual." [Online]. Available: http://la.epfl.ch/files/content/sites/la/files/shared/ common/Research/openbuild/Manual.pdf 